\title{
Enantioseparation of Neutral Compounds on a Quinine Carbamate-Immobilized Zirconia in Reversed-Phase Capillary Electrochromatography
}

\author{
Munrak Lee, Jurim Gwon, and Jung Hag Park* \\ Department of Chemistry, Yeungnam University, Gyeongsan 712-749, Korea. *E-mail: jhpark@ynu.ac.kr \\ Received October 27, 2009, Accepted November 6, 2009
}

\begin{abstract}
Quinine (QN) is a weak anion-exchange type chiral selector and QN-based silica stationary phases have been widely used for enantioseparation of acidic chiral analytes in HPLC and recently in CEC. In this work we report enantioseparation of non-acidic chiral analytes on a quinine carbamate-immobilized zirconia (QNZ) in reversed-phase (RP) CEC. Influences of $\mathrm{pH}$, composition of the buffer, acetonitrile content and the applied voltage on enantioseparation were examined. Enantiomers of the analytes investigated are well separated in acetonitrile/phosphate buffer mobile phases. Separation data on QNZ were compared to those on QN-bonded silica (QNS). Retention was longer but better enantioselectivity and resolution were obtained on QNZ than QNS.
\end{abstract}

Key Words: Enantioseparation, Neutral chiral compounds, Weak anion exchanger, Quiniune carbamateimmobilized zirconia, Capillary electrochromatography

\section{Introduction}

Capillary electrochromatography (CEC) is a hybrid technique that combines the useful features of capillary electrophoresis and high performance liquid chromatography (HPLC), and its high efficiency and fast analysis make the technique an attractive tool for chiral separation. ${ }^{1,2}$ Three types of stationary phase columns, open tubular capillary, monolithic capillary and packed capillary, are used for CEC. Despite such problems as bubble formation and zone broadening dueto end-frits in the packed capillaries, packed capillary columns are a widely used type of capillary format for CEC since existing HPLC stationary phases can be conveniently utilized.

Quinine $(\mathrm{QN})$ has been used widely for enantiomer separation in HPLC as a chiral ion-pairing agent in the mobile phases and as a chiral stationary phase (CSP) ligand. ${ }^{3-6}$ CSPs based on the use of carbamoylated derivatives of QN and quinidine as anionexchange type selectors were found to be highly stereoselective for the direct resolution of chiral acids in reversed-phase $\mathrm{LC}^{7-20}$ and CEC. ${ }^{21-27}$ Chiral recognition mechanism on carbamoyl QNimmobilized stationary phases in organic-aqueous buffer mobile phases involves simultaneously ion pairing, and differential hydrogen bonding (HB), $\pi-\pi$ and van der Waals interactions between carbamoyl QN and enantiomers of the chiral analyte. ${ }^{8,28}$ Ion pairing interaction is much stronger than the remaining stereoselective interactions and is essential in the chiral recognition processes. ${ }^{8,25,27}$ An example from an earlier work shows that an acidic chiral analyte ( $N$-3,5-dinitrobenzoyltryptophn) that is present in the form of anions at the $\mathrm{pH}$ of the mobile phase is more retained $\left(\mathrm{k}_{1}=19.13\right)$ and well separated $(\alpha=5.17)$ while its nonacidic derivative ( $N$-3,5-dinitrobenzoyltryptophn methyl ester) is much less retained $\left(\mathrm{k}_{1}=9.47\right)$ and not enantioseparated at all $(\alpha=1.00)^{8}$

On the 18-crown-6-2,3,11,12-tetracarboxylic acid-bonded CSPs, which have been widely used for the separation of primary amino compounds and the chiral recognition mechanism on it is known to require complexation interactions between pri- mary ammonium ions and crown oxygens, successful enantioseparations were obtained for secondary amines that do not

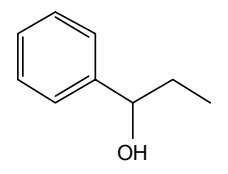

1-phenyl-1-propanol

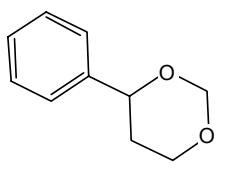

4-phenyl-1,3-dioxane strongly bind to crown ether oxygens, ${ }^{29}$ and for the analytes with no primary or secondary amino groups that can be protonated and undergo complexation interactions with the crown moiety. ${ }^{30}$ We thought it interesting to see if nonionic chiral compounds can be resolved on the QN-based CSP, in a similar vein to the enantioseparation of nonionic analytes on the crown ether CSPs. In this work we report enantioseparation of two neutral compounds, 1-phenyl-1-propanol and 4-phenyl-1,3-dioxane, on a carbamoyl QN-immobilized zirconia (QNZ) CSP in reversedphase CEC (RP-CEC). Although both compounds are not Brönsted acidic, 1-phenyl-1-propanol is HB acidic but 4-phenyl-1,3dioxane is not. ${ }^{31}$ It is interesting to see if the HB acidity may play a role in chiral resolution of the non-Brönsted acidic analytes on QNZ.

Zirconia as stationary phase support has received considerable attention over the last decade. ${ }^{32-34}$ Zirconia particles are very robust material; they show no detectable signs of dissolution over the $\mathrm{pH}$ range from 1 to 14 and have been used for prolonged periods at temperatures up to $200{ }^{\circ} \mathrm{C}$ in chromatographic separation. Zirconia-based stationary phases have been increasingly used in CEC separations recently. ${ }^{35-43}$ To our knowledge enantioseparation of non-acidic, neutral compounds on QNZ by RP-CEC has not been reported while QNZ was used for enantioseparation of acidic chiral compounds by RPLC. ${ }^{44-46}$ Influences of $\mathrm{pH}$, acetonitrile content, composition of the buffer and applied voltage on enantioseparation were examined. Enantioseparation data on QNZ were also compared to thoseon carbamoyl QN-bonded silica (QNS) to see if there are any differences 
in enantioseparation performance depending on the type of support material.

\section{Materials and Methods}

Materials. All reagents used for the preparation of the stationary phase were reagent grade or better. Quinine, anhydrous toluene, petroleum ether, $N, N$-dimethylformamide and anhydrous tetrahydrofuran were obtained from Aldrich (Milwaukee, USA). $n$-Hexane was purchased from EM Sciences (Gibbstown, USA). Zirconia (pore size, $30 \mathrm{~nm}$; particle diameter, $5 \mu \mathrm{m}$; specific surface area, $30 \mathrm{~m}^{2} / \mathrm{g}$; density, $2.40 \mathrm{~g} / \mathrm{mL}$ ) was obtained from ZirChrom Separations (Anoka, MN, USA). Silica (pore size, 30 $\mathrm{nm}$; particle diameter, $5 \mu \mathrm{m}$; specific surface area, $100 \mathrm{~m}^{2} / \mathrm{g}$; density, $0.45 \mathrm{~g} / \mathrm{mL}$ ) was obtained from Macherey-Nagel (Düren, Germany). Water was processed with an Elgastat UHQ water purification system (Bucks, UK). 1-Phenyl-1-propanol and 4phenyl-1,3-dioxane were obtained from Aldrich (Milwaukee, USA).

Preparation of quinine carbamate-immobilized zirconia and silica. Quinine 3-triethoxysilylpropylcarbamate was synthesized as previously reported ${ }^{8}$ and characterized by elemental analysis, IR and NMR spectroscopy. Three hundred mol amount of quinine 3-triethoxysilylpropylcarbamatewas dissolved in 4 $\mathrm{mL}$ of ethanol and the solution was added slowly to the slurry of $1 \mathrm{~g}$ of zirconia in $4 \mathrm{~mL}$ of water. The suspension was refluxed for $1 \mathrm{~h}$ and the particles were filtered and washed 2 times with $5 \mathrm{~mL}$ of $2 / 1(\mathrm{v} / \mathrm{v})$ ethanol/water and once with $3 \mathrm{~mL}$ of acetone. The particles were then dried in vacuum at $80^{\circ} \mathrm{C}$. Surface coverage of quinine 3-triethoxysilylpropylcarbamate adsorbed on the zirconia surface based on the percent carbon was found to be $0.58 \mu \mathrm{mol} \mathrm{m}^{-2}$. Quinine 3-triethoxysilylpropylcarbamate was bonded onto silica as previously reported. ${ }^{8}$ Surface coverage of the chiral selector based on the percent carbon was found to be $0.56 \mu \mathrm{mol} \mathrm{m}{ }^{-2}$.

Columns. QNZ and QNS were slurry-packed in $n$-hexane by using Alltech slurry packer (Deerfield, USA) at $c a .5000$ psi into $100 \mu \mathrm{m}$ i.d. $\times 363 \mu \mathrm{m}$ o.d. fused silica capillaries (Polymicro

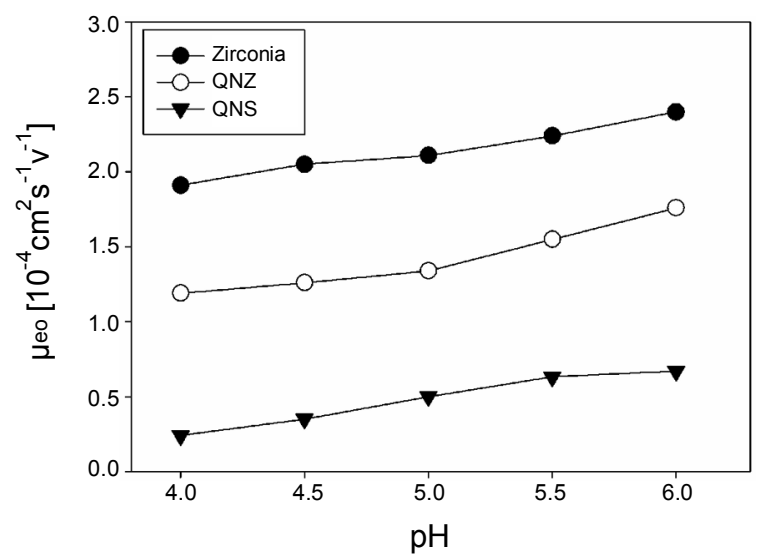

Figure 1. Variation of electroosmotic mobility $\left(\mu_{\mathrm{eo}}\right)$ with $\mathrm{pH}$. Conditions: column, length $35 \mathrm{~cm}$ (packed bed $20 \mathrm{~cm}$ ); EOF marker, thiourea; mobile phase, 5:95 (v/v\%) ACN/phosphate buffer (10 mM); applied voltage, $15 \mathrm{kV}$; temperature, $25^{\circ} \mathrm{C}$; injection, $10 \mathrm{kV}$ for $3 \mathrm{~s}$; detection, at $254 \mathrm{~nm}$.
Technologies, Phoenix, USA) with packed bed length of 200 $\mathrm{mm}$ and total length of $350 \mathrm{~mm}$. No attempt was made to optimize the packing procedure. Since it was not possible to sinter the zirconia particles to make frits, 5 - $\mu \mathrm{m}$ bare silica were introduced into the capillary for several centimeters both before and after packing with zirconia particles. Internal frits were made by sintering the silica particles at a distance of $3 \mathrm{~mm}$ from the beginning of the zirconia particle bed while water flowed through the column under a pressure of $1000 \mathrm{psi}$. The loose non-sintered silica particles were flushed away, and a detection window was made immediately after the outlet frit. ${ }^{47}$

Chromatography. CEC measurements were carried at $25^{\circ} \mathrm{C}$ on an Agilent $\mathrm{HP}^{3 \mathrm{D}} \mathrm{CE}$ system (Palo Alto, USA) with $\mathrm{HP}^{3 \mathrm{D}} \mathrm{CE}$ ChemStation software. An external pressure of 10 bars was applied to both buffer reservoirs. The mobile phases were mixtures of acetonitrile with sodium hydrogen phosphate buffer of varying composition. They were filtered through a nylon membrane filter of $0.2-\mu \mathrm{m}$ pore size and degassed prior to use. The columns were preconditioned with at least $12 \mathrm{~h}$ using the mobile phase before the chromatographic measurement. Samples dissolved in the mobile phase were injected electro-kinetically at $15 \mathrm{kV}$ for $5 \mathrm{~s}$ and detected at $254 \mathrm{~nm}$. Retention times of two consecutive injections were in agreement within 3\%. Dead time was measured by injecting thiourea.

\section{Results and Discussion}

Influence of pH. Zircanol groups on zirconia surface can undergo the following acid-base reactions and either positive or negative surface charge can develop depending on the $\mathrm{pH}$ of the mobile phase:

$$
\begin{aligned}
& \mathrm{ZrOH} \leftrightarrows \mathrm{ZrO}^{-}+\mathrm{H}^{+} \\
& \mathrm{ZrOH}_{2}^{+} \leftrightarrows \mathrm{ZrOH}+\mathrm{H}^{+}
\end{aligned}
$$

Zirconia possesses net zero charge at isoelectric point of $\mathrm{pH}$ 5 - 6 which can vary depending on the crystalline form and the material of synthesis. Thus the direction of EOF can be either cathodic above this $\mathrm{pH}$ or anodic below this $\mathrm{pH} \cdot{ }^{39,41}$ Figure 1 shows variation of electroosmotic mobilities $\left(\mu_{\mathrm{eo}}\right)$ measured by thiourea on bare zirconia, QNZ and QNS with $\mathrm{pH}$ in the 5:95 (v/v \%) ACN/phosphate buffer. ACN was chosen as the organic solvent as it usually gives a faster and better separation in CEC $^{25,27,48}$ Directions of EOF for both bare zirconia and QNZ are cathodic within the $\mathrm{pH}$ range from 4 to 6 . Phosphate ions as Lewis bases are able to bind strongly to the Lewis acid sites on zirconia surface to provide negative surface charges ${ }^{34}$ and hence cathodic EOF, regardless of the mobile phase $\mathrm{pH} .{ }^{43}$ Expression for $\mu_{\mathrm{eo}}$ in CE given by Smoluchowski $\left(\mu_{\mathrm{eo}}=\varepsilon \cdot \varepsilon_{\mathrm{o}} \cdot \zeta / \eta\right.$, where $\varepsilon$ is the dielectric constant of the medium, $\varepsilon_{0}$ is the permittivity of the vacuum, $\zeta$ is the zeta potential and $\eta$ is the viscosity of the medium) indicates that $\mu_{\mathrm{eo}}$ is only dependent on the $\zeta$ potential for a given medium. The $\zeta$ potential is proportional to the surface charge density. Magnitude of EOF for bare zirconia increases with $\mathrm{pH}$ as more zircanol groups dissociate according to eq (1) to increase surface charges, resulting in increasing EOF.

On the surface of QNZ present are zircanol and amino groups 


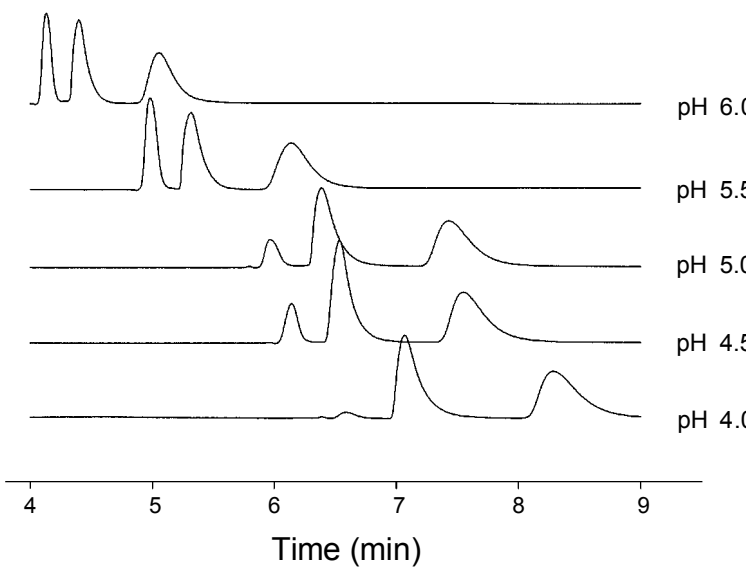

Figure 2. Chromatograms showing the enantioseparation of 1-phenyl1-propanol on QNZ at different $\mathrm{pH}$. The first-eluting peak is thiourea, co-injected with the analyte. Conditions are as specified in Fig. 1.
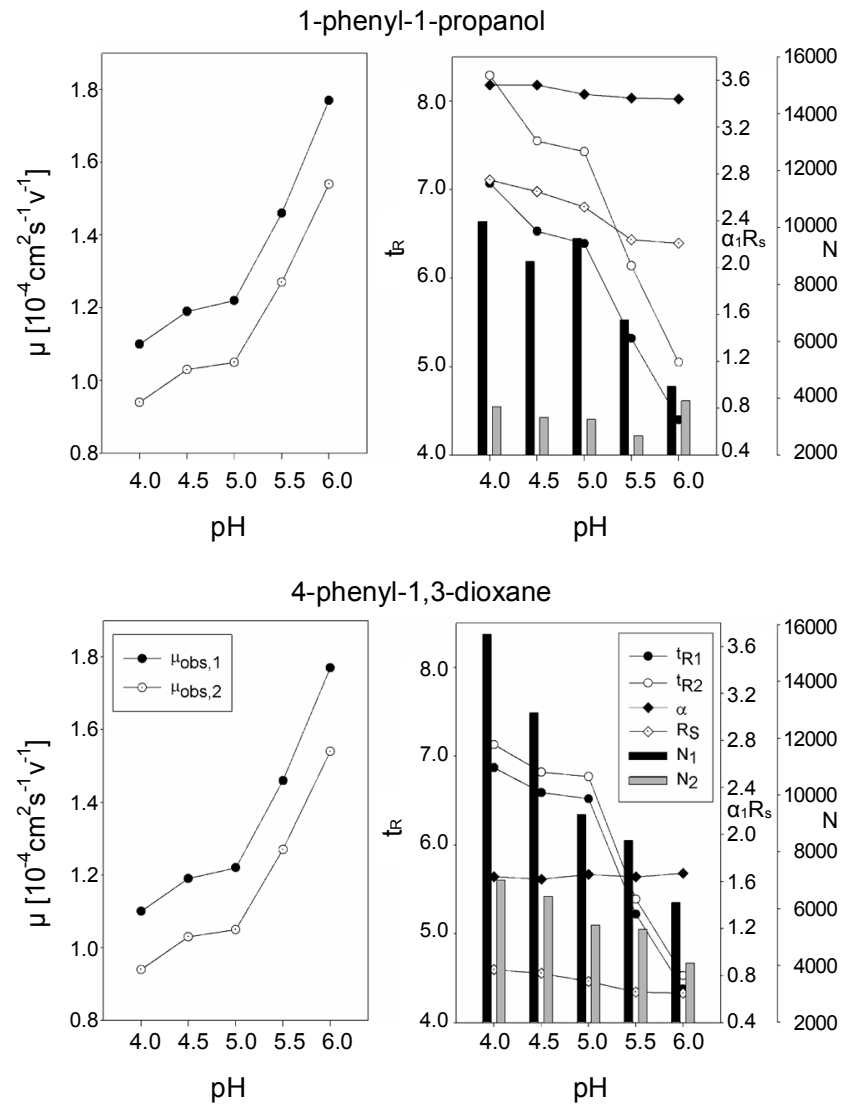

Figure 3. Influence of $\mathrm{pH}$ on observed mobilities $\left(\mu_{\mathrm{obs}}\right)$, retention times $\left(t_{\mathrm{R}}\right)$, enantioselectivity $(\alpha)$, resolution $\left(\mathrm{R}_{\mathrm{s}}\right)$ and theoretical plate numbers $(N)$ for 1-phenyl-1-propanol and 4-phenyl-1,3-dioxane on QNZ. Conditions are as specified in Fig. 1.

on quinuclidine ring with a $\mathrm{p} K$ of around 9.8 and quinoline ring with a $\mathrm{p} K$ of around 3.9. Amino groups on quinuclidine are fully protonated while those on quinoline are partially protonated in mobile phases of $\mathrm{pH}$ between 4.0 and 6.0. The net charge of the QNZ surface and thus the direction and magnitude of EOF will change with the mobile phase $\mathrm{pH}$ as opposite charges on the surface compensate each other and the resulting net charge determines the direction and magnitude of EOF. Cathodic EOF observed in the $\mathrm{pH}$ range studied indicates that the sum of negative charges from dissociated zircanol and adsorbed phosphate anions is greater than that of positive charges on protonated amino groups. Magnitude of EOF on QNZ increases monotonically with $\mathrm{pH}$ as more zircanol groups dissociate according to eq (1) to increase surface charges. The magnitude of EOF on QNZ is smaller than bare zirconia since the number of exposed dissociable zircanol groups is diminished as the surface is covered by adsorbed chiral selector.

Direction of EOF on QNS is cathodic in ACN-phosphate buffer mobile phases, which is in contradistinction with an earlier observation that EOF is anodic at $\mathrm{pH}$ values below $c a .6$ in methanol-acetate buffer mobile phases. ${ }^{25,27}$ It is very likely that the phosphate anions adsorb onto silica surface to a greater extent than acetate anions and the negative charges from adsorbed phosphate and dissociated silanol groups effectively cancel out the positive charges on amino groups of quinine moiety, leaving net negative charge on the surface. EOF on QNZ is greater than that on QNS. Estimated surface coverage is about the same for QNZ $\left(0.58 \mu \mathrm{mol} / \mathrm{m}^{2}\right)$ and QNS $\left(0.56 \mu \mathrm{mol} / \mathrm{m}^{2}\right)$. The concentration of surface hydroxyl groups for silica $\left(8-9 \mu \mathrm{mol} / \mathrm{m}^{2}\right.$ for fully hydroxylated silica $\left.{ }^{49}\right)$ and zirconia $\left(9.8 \mu \mathrm{mol} / \mathrm{m}^{2}\right.$ for maximally hydroxylated zirconia ${ }^{34}$ ) are not very different. It is likely that the extent of phosphate adsorption is greater on zirconia than on silica, yielding the greater net negative surface charge and hence greater EOF on QNZ than QNS.

Chromatogram for enantioseparation of 1-phenyl-1-propanol on QNZ in ACN/phosphate buffer of different $\mathrm{pH}$ is shown as an example in Fig. 2 , and observed mobilities $\left(\mu_{\mathrm{obs}}\right)$, retention time $\left(t_{R}\right)$, separation factor $(\alpha)$, resolution $\left(R_{s}\right)$ and theoretical plate numbers $(\mathrm{N})$ for 1-phenyl-1-propanol and 4-phenyl-1,3dioxane are plotted in Fig. 3. Retention decreases with the mobile phase $\mathrm{pH}$ as EOF increaseswith $\mathrm{pH}$. Enantioselectivity changes only slightly with $\mathrm{pH}$ as differential and additional intermolecular interactions of enantiomers of the neutral solute with the CSP are not to be affected strongly by the change in $\mathrm{pH}$. Resolution decreases with $\mathrm{pH}$ in general. The relationship, $R_{s}=1 / 4 N^{1 / 2} \cdot(\alpha-1)^{50}$ indicates that with $\alpha$ values essentially unvarying, decreasing plate numbers causes resolution to decrease.

Two processes are involved in the enantioseparation of chiral neutral solutes by CEC on a weak anion exchanger type CSP: EOF and interactions of the solute with the stationary phase. EOF is solely responsible for the migration of the neutral solute with no contribution from electrophoretic mobility. Chiral discrimination is based on the difference in binding strength of the analyte with CSP. For an acidic compound on QNZ, the binding is mainly driven by ionic interaction between the positively charged quinine moiety and the negatively charged analyte, and then van der Waals attractive and/or repulsive interactions including $\mathrm{HB}$ and $\pi-\pi$ interactions come to set off chiral discrimination of enantiomers. It is quite notable that neutral chiral compounds are quite well resolved on QNZ with no help of ionic interaction-driven binding to CSP. It seems that HB acidity plays a role in chiral resolution of the two non-Brönsted acidic analytes on QNZ. HB acidic 1-phenyl-1-propanol is longer retained and better resolved than HB basic 4-phenyl-1,3dioxane. 

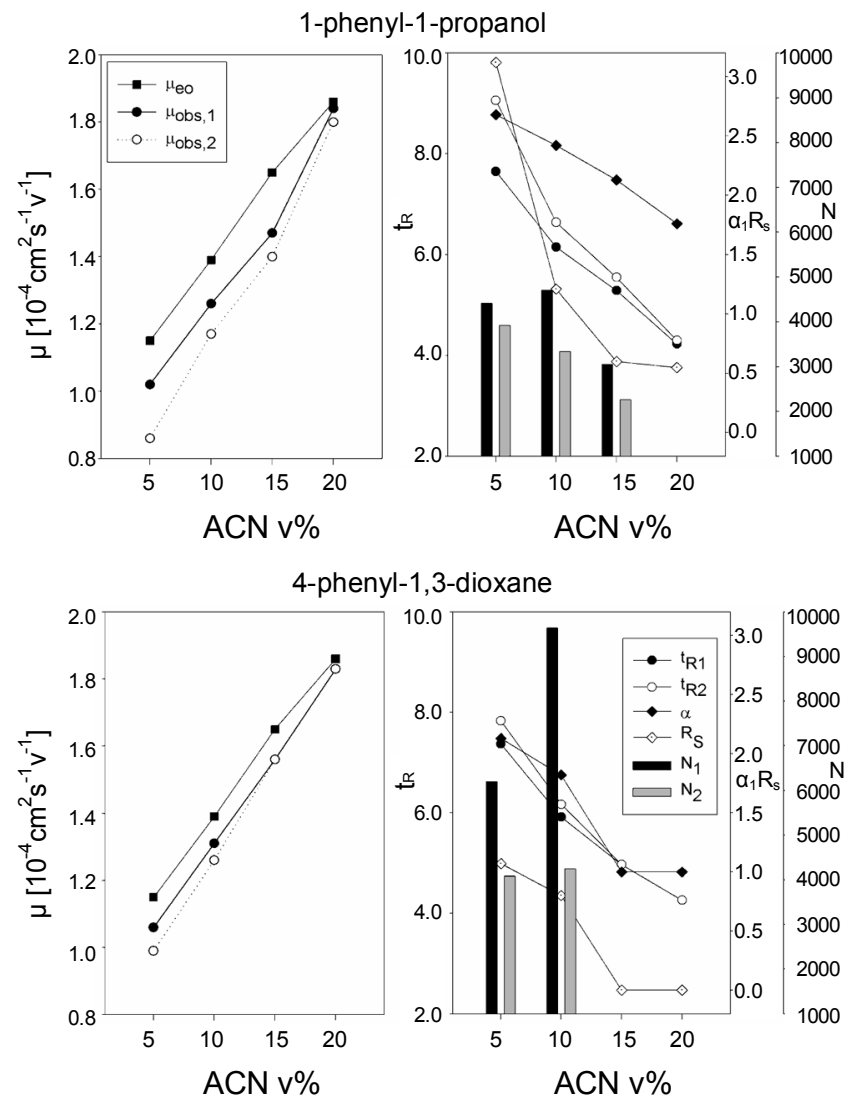

Figure 4. Influence of $\mathrm{ACN}$ content on electroosmotic mobility $\left(\mu_{\mathrm{eo}}\right)$, observed mobilities $\left(\mu_{\mathrm{obs}}\right)$, retention times $\left(t_{\mathrm{R}}\right)$, enantioselectivity $(\alpha)$, resolution $\left(\mathrm{R}_{\mathrm{s}}\right)$ and theoretical plate numbers $(N)$ for 1-phenyl-1-propanol and 4-phenyl-1,3-dioxane on QNZ. Mobile phase, ACN/phosphate buffer $(10 \mathrm{mM}, \mathrm{pH} 6.0)$. Other conditions are as specified in Fig. 1.

Influence of acetonitrile content, buffer concentration and applied voltage. Influence of the composition of ACN in the mobile phase on enantiomeric resolution of 1-phenyl-1-propanol and 4-phenyl-1,3-dioxane on QNZ was examined by varying volume percent of ACN from 5 to $20(\mathrm{v} / \mathrm{v} \%)$ in the ACN/ phosphate buffer $(10 \mathrm{mM})$ and separation data are plotted in Fig. 4. The electroosmotic mobilities increase while the $\varepsilon / \eta$ ratio computed using the $\varepsilon^{51}$ and $\eta^{52}$ data in the literature decreases slightly as ACN content is increased. The EOF is proportional to the $\varepsilon / \eta$ ratio of the medium in CE. This unexpected EOF behavior in CEC was also observed on a polymer-coated silica in $\mathrm{ACN} /$ ammonium acetate buffer solvents, ${ }^{53}$ indicating that the $\varepsilon / \eta$ dependence of EOF in CE is not applicable to CEC without limitation. The theoryof EOF in CEC is very complex due to a number of factors including the presence and inhomogeneity of the packing, duplex geometry of the column with packed and open segments and heterogeneity of the zeta potential, and thus the effect of these factors is likely responsible for deviations from the expected EOF behavior. ${ }^{54}$ Retention of the analytes decreases with increasing ACN content. On top of increasing EOF, the increasing amount of ACN also acts to increase the solubility of the analyte in the mobile phase and in turn decreases interaction with the stationary phase, yielding shortened retention. With increasing $\mathrm{ACN}$ content $R_{s}$ and $\alpha$ also decreases rapidly. 4-Phenyl-1,3-dioxane was not resolved in 15\% ACN. 1-

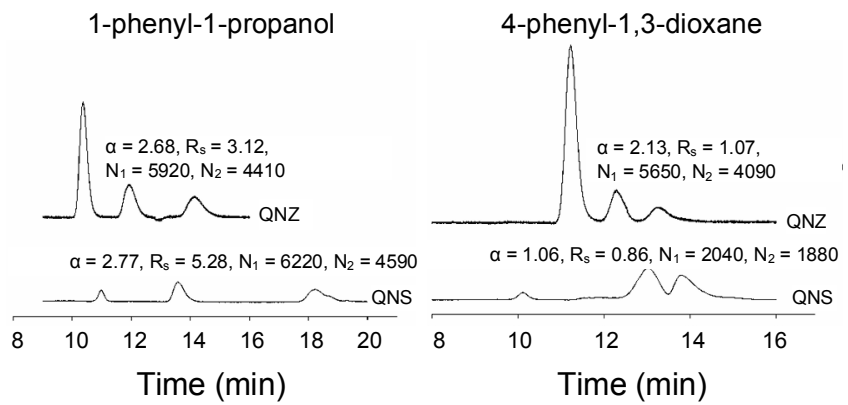

Figure 5. Chromatograms showing enantioseparation of 1-phenyl1-propanol and 4-phenyl-1,3-dioxane on QNZ and QNS. Applied voltage: QNZ, $10 \mathrm{kV}$; QNS, $15 \mathrm{kV}$. Other conditions are as specified in Fig. 4.

Phenyl-1-propanol was resolved in $15 \% \mathrm{ACN}$, but not resolved in $20 \% \mathrm{ACN}$.

As expected, EOF decreased with increasing buffer concentration as the double layer thickness and zeta potential decrease. Retention increased in general while enantioselectivity and resolution for the neutral analytes changed only slightly with buffer concentration. With increasing applied voltage EOF increased as expected and hence retention of the solutes decreased. Resolution, plate number and selectivity generally increased with voltage.

Comparison with QNS. Zirconia and silica particles used possess identical particle size and pore diameter, and QNZ and QNS, having about the same surface coverage, were packed into columns to have identical packed bed length under the identical packing conditions. Electric field strength was adjusted so that dead volume marker can be eluted with similar retention times on the two columns. Figure 5 shows chromatograms for separations of 1-phenyl-1-propanol and 4-phenyl-1,3-dioxane on QNZ and QNS in 5:95 (v/v \%) ACN/phosphate buffer. Even with a lower electric filed strength $(10 \mathrm{kV})$ retention is shorter on QNZ than on QNS with a higher electric filed strength $(15 \mathrm{kV})$. 1-Phenyl-1-propanol is better resolved on QNS than QNZ while 4-phenyl-1,3-dioxane is better resolved on QNZ than QNS.

\section{Conclusions}

Two neutral compounds, 1-phenyl-1-propanol and 4-phenyl1,3-dioxane, were enantioresolved on QNZ in RP-CEC. Influences of $\mathrm{pH}$, composition of ACN and buffer, and the applied voltage on enantioseparation were examined in $\mathrm{ACN} /$ phosphate buffer mobile phases. Enantiomers of the two analytes are well separated without participation of ion pairing interaction, which is nonstereoselective but thought to be essential in the chiral recognition processes. It is noted that HB acidic 1-phenyl-1propanol is longer retained and better resolved than HB basic 4-phenyl-1,3-dioxane. Separation data on QNZ were also compared to those on QNS. QNZ showed better enantioresolution of 4-phenyl-1,3-dioxane while QNS showed better results for 1-phenyl-1-propanol. Under the same electric filed strength QNZ shows a higher EOF, which reduces run times, and yet shows comparable separation efficiency to QNS. Work is in progress to enantioresolve more variegated non-acidic chiral compounds on QNZ. 
Acknowledgments. This work was supported in part by the Korea Research Foundation Grant funded by the Korean Government (MOEHRD) (KRF-2006-312-C00269) and in part by the Korea Science and Engineering Foundation (R01-2006000-10004-0).

\section{References}

1. Capillary Electrochromatography; J. Chromatogr. Library, Vol. 62, Deyl, Z., Svec, F., Eds.; Elsevier: Amsterdam, 2001.

2. Capillary Electrochromatography; Bartle, K. D., Meyers, P., Eds.; Royal Society of Chemistry Monographs, Royal Society of Chemistry: London, 2001.

3. Pettersson, C. J. Chromatogr. 1984, 316, 553.

4. Pettersson, C.; Schill, G. J. Liq. Chromatogr. 1986, 9, 269.

5. Rosini, C.; Altemura, P.; Pini, D.; Bertucci, C.; Zullino, G.; Salvadori, P. J. Chromatogr. 1985, 348, 79.

6. Salvadori, P.; Rosini, C.; Pini, D.; Bertucci, C.; Uccello-Barretta, G. Chirality 1989, $1,161$.

7. Lämmerhofer, M.; Lindner, W. GIT Special: Chromatogr. Int. 1996, $40,16$.

8. Lämmerhofer, M.; Lindner, W. J. Chromatogr. A 1996, 741, 33.

9. Lämmerhofer, M.; Maier, N. M. Lindner, W. Am. Lab. 1998, $30,71$.

10. Lämmerhofer, M.; Di Eugenio, P.; Molnar, I.; Lindner, W. J. Chromatogr. B 1997, 689, 123.

11. Mandl, A.; Nicoletti, L.; Lämmerhofer, M.; Lindner, W. J. Chromatogr. A 1999, 858, 1.

12. Kleidernigg, O. P.; Lämmerhofer, M.; Lindner, W. Enantiomer 1996, $1,387$.

13. Piette, V.; Lämmerhofer, M.; Bischoff, K.; Lindner, W. Chirality 1997, 9, 157

14. Piette, V. Ph.D. Thesis, Facultes Universitaires Notre-Damede la Paix, Namur. 1999.

15. Franco, P.; Lämmerhofer, M.; Klaus, P.; Lindner, W. J. Chromatogr. A 2000, 869, 111.

16. Franco, P.; Klaus, P.; Minguillon, G.; Lindner, W. Chirality 2001, $13,177$.

17. Peter, A.; Torok, G.; Toth, G.; Lindner, W. J. High Resolut. Chromatogr. 2000, 23, 628.

18. Peter, A. J. Chromatogr. A 2002, 955, 141.

19. Piette, V.; Lämmerhofer, M.; Lindner, W.; Crommen, J. J. Chromatogr. A 2003, 987, 421.

20. Krawinkler, K. H.; Maier, N. M.; Ungaro, R.; Sansone, F.; Casnati, A.; Lindner, W. Chirality 2003, 15, S15.

21. Preinerstorfer, B.; Bicker, W.; Lindner, W.; Lämmerhofer, M. J. Chromatogr. A 2004, 1044, 187.

22. Lämmerhofer, M.; Tobler, E.; Zarbl, E.; Lindner, W.; Svec, F.; Frechet, J. M. J. Electrophoresis 2003, 24, 2986.

23. Munoz, O. L. S.; Hernandez, E. P.; Lämmerhofer, M.; Lindner, W.; Kenndler, E. Electrophoresis 2003, 24, 390.

24. Tobler, E.; Lämmerhofer, M.; Mancini, G.; Lindner, W. Chirality 2001, 13, 64
25. Lämmerhofer, M.; Tobler, E.; Lindner, W. J. Chromatogr. A 2000 , 887,421

26. Lämmerhofer, M.; Lindner, W. J. Chromatogr. A 1999, 839, 167.

27. Lämmerhofer, M.; Lindner, W. J. Chromatogr. A 1998, 829, 115.

28. Maier, N. M.; Nicoletti, L.; Lämmerhofer, M.; Lindner, W. Chirality 1999, 11, 522.

29. Steffeck, R. J.; Zelechonok, Y.; Gahm, K. H. J. Chromatogr. A 2002, 947, 301.

30. Hyun, M. H.; Tan, G.; Xue, J. Y. J. Chromatogr. A 2005, 1097, 188.

31. Kamlet, M. J.; Abboud, J. L. M.; Taft, R. W. Prog. Phys. Org. Chem. 1981, 13, 485.

32. Nawrocki, J.; Dunlap, C. J.; Carr, P. W.; Blackwell, J. A. Biotechnol. Prog. 1994, 10, 561.

33. Jackson, P. T.; Carr, P. W. Chemtech 1998, 28, 29.

34. Nawrocki, J.; Rigney, M. P.; McCormick, A.; Carr, P. W. J. Chromatogr. A 1993, 657, 229.

35. Xie, M.-J.; Feng, Y.-Q.; Da, S.-L.; Meng, D.-Y.; Ren, L.-W. Anal. Chim. Acta 2001, 428, 255.

36. Xia, D.; Feng, Y.-Q.; Da, S.-L. J. Liq. Chromatogr. Rel. Technol. 2001, 24, 1881.

37. Tsuda, T.; Kitagawa, S.; Munesue, M. JP 2001235459 (2001).

38. Crosnier de Bellaistre, M.; Mathieu, O.; Randon, J.; Rocca, J.-L. J. Chromatogr. A 2002, 971, 199.

39. Randon, J.; Crosnier de Bellaistre, M.; Rocca, J.-L. Chromatographia 2003, 57, S/355.

40. Randon, J.; Crosnier de Bellaistre, M.; Rocca, J.-L. Actualite Chim. $\mathbf{2 0 0 5}, 283,36$

41. Randon, J.; Huguet, S.; Piram, A.; Puy, G.; Demesmay, C.; Rocca, J.-L. J. Chromatogr. A 2006, 1109, 19.

42. Crosnier de Bellaistre, M.; Randon, J.; Rocca, J.-L. Electrophoresis 2006, 27, 736 .

43. Shi, Z.-G.; Feng, Y.-Q.; Xu, L.; Zhang, M.; Da, S.-L. Talanta 2004, 63, 593.

44. Kim, I. W.; Kwon, S. H.; McNeff, C. V.; Carr, P. W.; Jang, M. D.; Park, J. H. Bull. Korean Chem. Soc. 2006, 27, 589.

45. Park, J. H.; Lee, J. W.; Kwon, S. H.; Cha, J. S.; Carr, P. W.; McNeff, C. V. J. Chromatogr. A 2004, 1050, 151.

46. Park, J. H.; Lee, J. W.; Song, Y. T.; Ra, C. S.; Cha, J. S.; Ryoo, J. J.; Lee, W.; Kim, I. W.; Jang, M. D. J. Sep. Sci. 2004, 27, 977.

47. Xiang, Y.; Yan, B.; McNeff, C. V.; Carr, P. W.; Lee, M. L. J. Chromatogr. A 2003, 1002, 71.

48. Krause, K.; Girod, M.; Chankvetadze, B.; Blaschke, G. J. Chromatogr. A 1999, 837, 51.

49. Unger, K. K. Porous Silica; J. Chromatogr. Library, Vol. 16, Elsevier: Amsterdam, 1979.

50. Bowser, M.; Bebault, G. M.; Peng, X.; Chen, D. D. Y. Electrophoresis 1997, 18, 2928.

51. Gagliardi, L. G.; Castells, C. B.; Ràfols, C.; Rosés, M.; Bosch, E. J. Chem. Eng. Data 2007, 52, 1103.

52. Thompson, J. W.; Kaiser, T. J.; Jorgenson, J. W. J. Chromatogr. A 2006, 1134, 201

53. Krause, K.; Chankvetadze, B.; Okamoto, Y.; Blaschke, G. J. Microcol. Sep. 2000, 12, 398.

54. Rathore, A. S. Electrophoresis 2002, 23, 3827. 